\title{
Energy-aware Routing to Maximize Lifetime in Wireless Sensor Networks with Mobile Sink
}

\author{
Ioannis Papadimitriou and Leonidas Georgiadis
}

\begin{abstract}
In this paper we address the problem of maximizing the lifetime in a wireless sensor network with energy and power constrained sensor nodes and mobile data collection point (sink). Information generated by the monitoring sensors needs to be routed efficiently to the location where the sink is currently located across multiple hops with different transmission energy requirements. We exploit the capability of the sink to be located in different places during network operation in order to maximize network lifetime. We provide a novel linear programming formulation of the problem. We show that maximum lifetime can be achieved by solving optimally two joint problems: a scheduling problem that determines the sojourn times of the sink at different locations, and a routing problem in order to deliver the sensed data to the sink in an energy-efficient way. Our model provides the optimal solution to both of these problems and gives the best achievable network lifetime. We evaluate numerically the performance of our model by comparing it with the case of static sink and with previously proposed models that focus mainly on the sink movement patterns and sojourn times, leaving the routing problem outside the linear programming formulation. Our approach always achieves higher network lifetime, as expected, leading to a lifetime up to more than twice that obtained with models previously proposed as the network size increases. It also results in a fair balancing of the energy depletion among the sensor nodes. The optimal lifetime provided by the theoretical analysis of our model can be used as a performance measure in order to test the efficiency of other heuristics that might be proposed in the future for a practical implementation of a real system. Our formulation can also be used as a starting point on which new algorithms can be implemented.
\end{abstract}

Index Terms-Wireless Sensor Networks, Lifetime Maximization, Energy-aware Routing, Mobile Sink, Linear Programming

\section{INTRODUCTION}

The field of wireless multi-hop networks has attracted significant attention by many researchers recently because of its large number of new and exciting applications [1], [2]. Inside this field, wireless sensor networks play a special role in home automation, environmental monitoring, military, health, and other commercial applications. A sensor network is composed

A preliminary version of this work appeared in the Proceedings of $13^{\text {th }}$ International Conference on Software, Telecommunications and Computer Networks (SoftCOM 2005 - Symposium on Future Wireless Systems), Marina Frapa - Split, Croatia, September 2005. Manuscript received November 10, 2005; revised February 28, 2006, and April 30, 2006.

I. Papadimitriou was partially supported for this work by the Public Benefit Foundation “ALEXANDER S. ONASSIS”, Athens, GREECE.

I. Papadimitriou and L. Georgiadis are with Division of Telecommunications, Department of Electrical and Computer Engineering, Aristotle University of Thessaloniki, Thessaloniki 541 24, GREECE.

E-mails: ipapad@egnatia.ee.auth.gr (corresponding author), leonid@auth.gr . of a large number of small low-cost sensor nodes, which are typically densely and randomly deployed either inside the area in which a phenomenon is being monitored or very close to it. The sensor nodes, which consist of sensing, data processing, and communicating components, gather information about the physical world and communicate unattended in short distances. One or more data collection points (sinks), either static or mobile, have the responsibility of collecting the information gathered by the sensors for further processing or making decisions based on the observations and performing appropriate actions. The special constraints and technical challenges that arise because of the unique characteristics of sensing devices pose many new problems and issues that have to be addressed when designing a wireless sensor network [3], [4], [5]. Such an issue is the efficient management of the finite amount of energy provided by the battery-operated sensor nodes.

In this paper we focus on the problem of maximizing the lifetime of a wireless sensor network where the sensor nodes communicate with the sink by delivering the sensed data across multiple hops with different transmission energy requirements. That is, there is flexibility of transmitter power adjustment and the energy consumption rate per unit information transmission is not the same for all neighbors of a sensor, but depends on the choice of the next hop node. The lifetime of the network is defined as the time until a sensor node drains out of battery energy for the first time, a definition commonly used in the literature.

Although the problem of maximum lifetime routing has been studied extensively (see Section II for references to prior work), most of the previous approaches assume static data collection points (sinks) and focus on the problem of selecting energy-efficient routing paths to prolong network lifetime. However, some recent papers have started to explore the idea of exploiting the mobility of the sink for the purpose of collecting the information from the sensors in a more efficient and reliable manner.

In our setup, the sensors are realistically randomly deployed in the field (their placement does not rely on any specific pattern, e.g., grid network). The sink is mobile and can move to different places during network operation (the sensors' locations and possible sink locations are not necessarily the same). The problem that arises is, for how long the sink must stay at each place and how the sensors must deliver their data to the sink during its sojourn time at a given location, in order to maximize network lifetime. We show that maximum network lifetime can be achieved by solving optimally these 
two joint problems: the scheduling problem that determines the sink sojourn times, and the routing problem to find the appropriate energy-efficient paths. We show in Section III-B that these two optimization problems can be written as a linear programming model, capable of expressing network lifetime in terms of sink sojourn times at possible locations. Our model provides the optimal solution to both of these problems and gives the best achievable lifetime.

Numerical results for various networks with different sizes and sink node placements are presented in Section IV. We evaluate through simulations the performance of our model by comparing it with the case of static sink and with previously proposed models that focus mainly on the sink movement patterns and sojourn times, leaving the routing problem outside the linear programming formulation. The main performance metric of interest is the duration of network operation before a sensor node drains out of battery energy for the first time. Our approach always achieves higher network lifetime, as expected, leading to a lifetime up to more than twice that obtained with models previously proposed as the network size increases. It also results in a fair balancing of the energy depletion among the sensor nodes.

Our linear programming formulation reveals the necessity to develop new heuristic algorithms that take into account at the same time both of these optimization problems (the scheduling and the routing problem). Such algorithms can be used online in an adaptive and distributed environment where the sink sojourn times are not determined a priori. In any case, we note that the optimal lifetime provided by the theoretical analysis of our model can be used as a performance measure in order to test the efficiency of other heuristics that might be proposed in the future. Our formulation can also be used as a starting point on which new algorithms can be implemented. These issues are discussed in Section V.

The rest of paper is organized as follows. In Section II we give some references to prior work related to the problem of maximum lifetime routing and exploiting the mobility of data collection points (sinks). Section III provides formal definitions of the wireless sensor network model and formulation of the problem as a linear program. Numerical results are presented in Section IV, while in Section V we discuss some interesting issues for further study. Finally, Section VI summarizes the conclusions of our work. In the Appendix, we present a generalization of our problem formulation which can be useful in certain cases.

\section{RELATED WORK}

The problem of maximum lifetime routing in wireless sensor networks has received significant attention. In the work by Chang and Tassiulas [6], [7], [8], [9], the information obtained by the monitoring sensors needs to be routed in an energyefficient way to a set of static designated gateway nodes. The routing problem is formulated as a linear programming problem and a shortest cost path routing algorithm is proposed. Two different models are considered for the information generation processes (constant information generation rates and arbitrary information generation processes). It is shown that the proposed algorithm can achieve network lifetime that is very close to the optimal lifetime obtained by solving the linear program.

Other energy-aware routing algorithms in networks with lifetime requirements are proposed in [10], [11], [12], [13], [14]. In [10] the maximum lifetime data gathering and aggregation problem in wireless sensor networks is considered. The experimental results demonstrate that the proposed algorithms significantly outperform previous methods in terms of system lifetime. In [11] the problem of rate allocation is investigated for sensor networks under a given network lifetime requirement. A routing mechanism to prolong network lifetime is proposed in [12], where each node adjusts its transmission power to send data to its neighbors. In [13] an energy-aware approach for routing delay-constrained data is proposed. The approach finds energy-efficient paths for real-time data subject to end-to-end delay requirements. The energy conserving routing problem is formulated in [14] as a nonlinear program. It is proved that the nonlinear program can be converted to an equivalent maximum multi-commodity concurrent flow problem and an iterative approximation algorithm is developed based on a revised shortest path scheme.

The problem of efficiently positioning the data collection points (sinks) in a wireless sensor network is addressed in [15], [16]. In [15] it is shown that the choice of positions has a marked influence on the data rate, or equivalently, the power efficiency of the network. In [16] multiple sinks are used not only to increase the manageability of the network, but also to reduce the energy dissipation at each node.

Some of the recent works that exploit the mobility of sinks using a different approach than ours are presented in [17], [18], [19], [20], [21], [22]. In [17] power saving is achieved using predictable observer mobility in a single hop communication network. A scalable energy-efficient asynchronous dissemination protocol is presented in [18] to minimize energy consumption in both building a near-optimal dissemination tree and disseminating data to mobile sinks. The authors in [19] present an integer linear programming model to determine the locations of multiple sinks and a flow-based routing protocol is used. In [20], [21] a learning-based approach is proposed to efficiently and reliably route data to a mobile sink, where the sensors in its vicinity learn the movement pattern over time and statistically characterize it as a probability distribution function. Instead of using multi-hop routing, the authors in [22] consider a clustered network where each sensor sends the information to its cluster head and a mobile collector visits every cluster head according to a schedule to collect the data. Although routing is simpler in this case, since it does not depend on sink location, the model in [22] may result in higher implementation cost and complexity in order to organize the network into clusters and assign every sensor to a cluster head. Furthermore, if the information gathered by the sensors is crucial and delay-sensitive (fire detection, intrusion alarm, pressure increase), then this approach may turn out to be ineffective, since there is a significant delay for the collector to visit every cluster head. In our setup however, the information is acquired by the sink almost at the moment that it is created. 
In any case, if time limitations are not strict and the cost of organizing the network into clusters is relatively small, the approach in [22] is reasonable and can be used as an alternative to our model.

The work closest to ours is the one presented in [23]. A linear programming formulation is given for the problem of determining the sink sojourn times at different points in the network that induce the maximum network lifetime. However, the authors in [23] consider only a very special case of wireless sensor networks where the homogeneous sensor nodes are arranged in a bi-dimensional square grid composed of samesize cells. The initial amount of energy and the rate at which data packets are generated are the same for all sensors. The wireless channel is considered bi-directional and symmetric. There is no power control since the transmission range is the same for all sensor nodes (equal to the size of a cell of the grid), and the sensor locations and the possible sink locations are the same. Differently from our approach, the model in [23] determines only the sink movement patterns and sojourn times, leaving the routing problem outside the linear programming formulation. The shortest path algorithm used to route the packets to the sink during its sojourn time at a given location, although energy-aware, does not take into account the remaining energy of the sensors, thus resulting in an overall network lifetime which is not optimal.

\section{DEFINITIONS AND PROBlem Formulation}

\section{A. Wireless Sensor Network Model}

The wireless sensor network consists of a set $N$ of sensor nodes and a sink node $s$ collecting the information from the sensors. After having been randomly deployed in the sensor field, the sensors remain stationary at their initial locations and continuously monitor the physical environment where they have been placed. Hence, there is a constant information generation rate $Q_{i}>0$ at every sensor node $i \in N$ (not necessarily the same for every sensor). On the contrary, the sink is mobile and can be found in different random places during network operation (the sensors' locations and possible sink locations are not necessarily the same). Let $L$ be the set of locations where the sink node can be and $t_{l} \geq 0$ the sojourn time at location $l \in L$, i.e., the total time the sink spends at location $l$. For the analysis in the next sections, we assume that the traveling time of the mobile sink from one location to another is small and thus can be neglected ${ }^{1}$ as in [23].

Sensor nodes communicate with the sink during its sojourn time at a given location by delivering the sensed data across multiple hops. That is, for a given location $l \in L$, the sink is not necessarily within the transmission range of every sensor node. Let $S_{i}^{l} \subseteq N \cup\{s\}$ be the set of nodes (either sensors or the sink) that are in the transmission range of sensor $i \in N$ for a given location $l \in L$ of the sink. If $j \in S_{i}^{l}$, then $j$ is called a

\footnotetext{
${ }^{1}$ Another approach is to assume that there are two mobile sinks, such that one of them is at the current location and the other one is placed at the location to be considered next. Hence, there is no additional time spent between two consecutive locations. Alternatively, if the sensor nodes have enough buffer capacity, they can store their sensed data and wait until the sink node arrives at the new location.
}

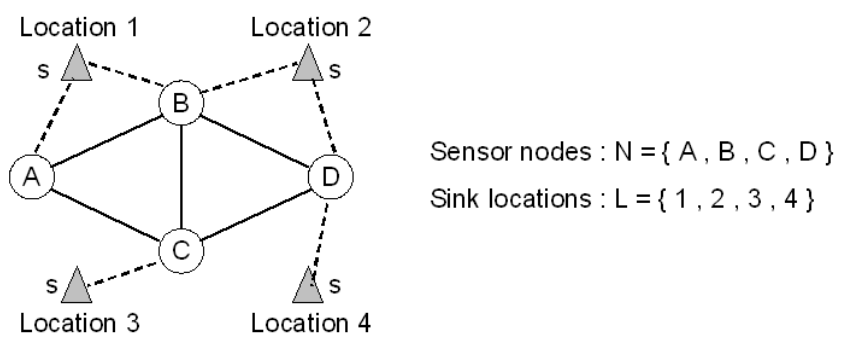

Permanent link between two sensor nodes

Link between a sensor and sink, existing only when the sink is at the corresponding location

Fig. 1. Sensor nodes communicate with the sink during its sojourn time at a location by delivering the sensed data across multiple hops.

neighboring node of $i$ for location $l$. Note that the only element that may be different among two sets $S_{i}^{l_{1}}, S_{i}^{l_{2}}, l_{1}, l_{2} \in L$, is the sink $s$, since the rest of the network (consisting of all sensors) remains static.

Consider for example the wireless sensor network shown in Fig. 1. Sensor nodes $B$ and $C$ are in the transmission range of $A$, regardless where the sink node is located. When $s$ is placed at location 1 , sensor $A$ can also communicate with $s$. Therefore, the set of neighboring nodes of sensor $A$ for location 1 is $S_{A}^{1}=\{B, C, s\}$. However, when $s$ moves to locations $2,3,4$, it is not in the transmission range of sensor $A$. Hence, $S_{A}^{2}=S_{A}^{3}=S_{A}^{4}=\{B, C\}$. Similarly for another sensor node, say node $C$, we have that $S_{C}^{1}=S_{C}^{2}=S_{C}^{4}=$ $\{A, B, D\}$, while $S_{C}^{3}=\{A, B, D, s\}$.

Every sensor $i \in N$ has an initial amount of battery energy $E_{i}>0$. The sink has no energy constraint, since it is typically a special node (different than the sensors) with plenty of battery energy which can also be easily renewed. The transmission energy consumed at sensor $i$ to transmit a data unit to its neighboring node $j$ is denoted by $e_{i j}^{T}>0$ and the energy consumed for reception by the receiver $j$ is denoted by $e_{i j}^{R}>0$. Note that the energy expenditure for an information unit transmitted by sensor $i$ depends on the next hop node and is not necessarily the same for every neighbor $j$. These quantities are network parameters determined by the energy dissipation model used. They usually depend on the physical distance between the nodes and other environmental and technological factors which are not addressed here in detail. In any case, we note that our problem formulation that follows does not depend on the energy consumption model used.

The above description of the wireless sensor network indicates that in order to transfer the information from the sensors to the sink, two complementary algorithms are necessary:

- a scheduling algorithm that determines for every location $l \in L$ the duration $t_{l}$ for which the sink node stays at that place,

- a routing algorithm to find the appropriate energy-efficient paths from every sensor node to the sink, for every location $l \in L$ for which $t_{l}>0$. 
TABLE I

SUMMARY OF NOTATION

\begin{tabular}{|l||l|}
\hline Notation & Definition \\
\hline \hline$N$ & Set of sensor nodes in the network. \\
\hline$L$ & Set of locations where the sink node $s$ can be placed. \\
\hline$t_{l}$ & Sink sojourn time at location $l \in L$. \\
\hline$Q_{i}$ & Information generation rate at sensor node $i \in N$. \\
\hline$E_{i}$ & Initial amount of battery energy at sensor node $i \in N$. \\
\hline$S_{i}^{l}$ & Set of neighboring nodes (either sensors or the sink) of sensor $i \in N$ for location $l \in L$. \\
\hline$e_{i j}^{T}$ & Energy consumed at sensor $i$ to transmit a data unit to its neighboring node $j \in S_{i}^{l}$. \\
\hline$e_{i j}^{R}$ & Energy consumed at sensor $j$ to receive the data unit transmitted by sensor $i$. \\
\hline$q_{i j}^{l}$ & Rate at which information is transmitted from sensor $i$ to its neighboring node $j$ during time $t_{l}$. \\
\hline$q_{i j}^{\text {max }}$ & Maximum possible rate at which information can be transmitted from sensor $i$ to its neighboring node $j$. \\
\hline$P_{i}$ & Maximum power that sensor node $i \in N$ can spend for every sink location. \\
\hline$\widehat{q}_{i j}^{l}$ & Amount of information transmitted from sensor $i$ to its neighboring node $j$ during time $t_{l}$. \\
\hline
\end{tabular}

Since the sink node can be found in different places during network operation, the decision of the routing algorithm depends on the location of the sink. Let $q_{i j}^{l}$ be the rate at which information is transmitted from sensor $i$ to its neighboring node $j$, to be assigned by the routing algorithm during time $t_{l}$. For each $q_{i j}^{l}$, there is a constraint $q_{i j}^{l} \leq q_{i j}^{\max }$, where $q_{i j}^{\max }$ is the maximum possible rate at which information can be transmitted from $i$ to $j$. These bounds can be viewed as link capacity constraints determined by the network environment. In addition to these link constraints, there is a power constraint for every sensor node. That is, for every sink location $l \in L$, the power expenditure at sensor $i \in N$ incurred by the transmissions and receptions of $i$ during time $t_{l}$ cannot exceed a maximum value $P_{i}$. This value can reflect the limitations imposed by the hardware of the sensors (transceiver circuitry, power unit, etc.).

The overall objective is to maximize the duration of network operation before a sensor node drains out of battery energy for the first time. In our model, the network lifetime is equal to the sum of the sojourn times of the sink at all possible locations (see Section III-B below). The sojourn times are constrained by the fact that the total energy consumed by each sensor node when the sink stays at different locations cannot exceed the sensor's initial amount of energy. Furthermore, the constraints on transmission rates and the power of sensors are also taken into consideration. We summarize in Table I the notation most commonly used in this paper for reader convenience. The last quantity in the table is introduced in the following section.

Note 1: The above description implies that we look at the system from the network layer perspective, i.e., the effect of the MAC layer and the related interference is not addressed here directly. This can be considered as a valid working assumption, an approach followed by many previous works in order to reduce the problem formulation complexity. However, we note that some of the implications imposed by layers lower than the network layer can be incorporated into our model. For example, in a high interference environment the energy expenditures $e_{i j}^{T}$, $e_{i j}^{R}$ in general will become "worse” (increase) in order to reflect the fact that more energy is required for communication. The signal-to-interference ratio requirements in a congested network can also be incorporated into the link constraints $q_{i j}^{\max }$, since a smaller value of this bound will constrain the maximum transmission power from sensor $i$ to its neighbor $j$ and, thus, control the interference to other sensors.

\section{B. Linear Programming Formulation}

In the following we show that our optimization problem can be written as a Linear Programming (LP) problem [24]. We disregard any specific details of battery technology and assume that the power and, consequently, energy expenditures at the sensors are directly proportional to the rate at which information is transmitted/received. Given the sink sojourn times $t_{l}$ and the information transfer rates $q_{i j}^{l}, i \in N, j \in S_{i}^{l}$, $l \in L$, the power (energy per time unit) consumed at sensor node $i$ when the sink is placed at location $l$ is given by

$$
\sum_{j \in S_{i}^{l}} e_{i j}^{T} q_{i j}^{l}+\sum_{j: i \in S_{j}^{l}} e_{j i}^{R} q_{j i}^{l},
$$

while the corresponding energy consumption for time duration $t_{l}$ is given by

$$
\left(\sum_{j \in S_{i}^{l}} e_{i j}^{T} q_{i j}^{l}+\sum_{j: i \in S_{j}^{l}} e_{j i}^{R} q_{j i}^{l}\right) t_{l} .
$$

The total energy consumed at sensor $i$ during network operation is the sum of the quantities in (2) over all locations $l \in L$, that is,

$$
\sum_{l \in L} \sum_{j \in S_{i}^{l}} e_{i j}^{T} q_{i j}^{l} t_{l}+\sum_{l \in L} \sum_{j: i \in S_{j}^{l}} e_{j i}^{R} q_{j i}^{l} t_{l} .
$$

The network lifetime defined as the length of time until the first battery drain-out among all sensor nodes in $N$, can also be expressed as the sum of the sojourn times of the sink at all possible locations,

$$
\sum_{l \in L} t_{l} .
$$


Our goal is to find the sink sojourn times $t_{l}$ and the information transfer rates $q_{i j}^{l}, i \in N, j \in S_{i}^{l}, l \in L$, that maximize the lifetime of the network under the flow conservation condition and under the constraint that the total energy consumed by each sensor node when the sink stays at different locations cannot exceed the sensor's initial amount of energy. Moreover, the constraints on transmission rates and the power expenditures of sensors must also be taken into account. From the above definitions, the problem of maximizing the overall network lifetime can be written as follows:

$$
\begin{gathered}
\text { Maximize } \sum_{l \in L} t_{l} \text { subject to } \\
t_{l} \geq 0, l \in L, \\
0 \leq q_{i j}^{l} \leq q_{i j}^{\max }, i \in N, j \in S_{i}^{l}, l \in L, \\
\sum_{j \in S_{i}^{l}} e_{i j}^{T} q_{i j}^{l}+\sum_{j: i \in S_{j}^{l}} e_{j i}^{R} q_{j i}^{l} \leq P_{i}, i \in N, l \in L, \\
\sum_{l \in L} \sum_{j \in S_{i}^{l}} e_{i j}^{T} q_{i j}^{l} t_{l}+\sum_{l \in L} \sum_{j: i \in S_{j}^{l}} e_{j i}^{R} q_{j i}^{l} t_{l} \leq E_{i}, i \in N, \\
\sum_{j: i \in S_{j}^{l}} q_{j i}^{l}+Q_{i}=\sum_{j \in S_{i}^{l}} q_{i j}^{l}, i \in N, l \in L
\end{gathered}
$$

Note that the flow conservation condition in (10) applies to each location $l \in L$ separately. That is, for every location of the sink, the total incoming information transfer rate plus the information generation rate at a sensor node equals the total outgoing information transfer rate from the sensor. Since the sink node $s$ is the only destination of the data flows generated by the sensors, it holds by definition that

$$
q_{s j}^{l}=0, j \in N, l \in L, \quad \text { and } \quad Q_{s}=-\sum_{i \in N} Q_{i} .
$$

By defining $\widehat{q}_{i j}^{l}=q_{i j}^{l} t_{l}$ as the amount of information transmitted from sensor $i$ to its neighboring node $j$ during time $t_{l}$, the optimization problem becomes:

$$
\begin{gathered}
\text { Maximize } \sum_{l \in L} t_{l} \text { subject to } \\
t_{l} \geq 0, l \in L, \\
0 \leq \widehat{q}_{i j}^{l} \leq q_{i j}^{\max } t_{l}, \quad i \in N, j \in S_{i}^{l}, l \in L, \\
\sum_{j \in S_{i}^{l}} e_{i j}^{T} \widehat{q}_{i j}^{l}+\sum_{j: i \in S_{j}^{l}} e_{j i}^{R} \widehat{q}_{j i}^{l} \leq P_{i} t_{l}, i \in N, l \in L, \\
\sum_{l \in L} \sum_{j \in S_{i}^{l}} e_{i j}^{T} \widehat{q}_{i j}^{l}+\sum_{l \in L} \sum_{j: i \in S_{j}^{l}} e_{j i}^{R} \widehat{q}_{j i}^{l} \leq E_{i}, i \in N, \\
\sum_{j: i \in S_{j}^{l}} \widehat{q}_{j i}^{l}+Q_{i} t_{l}=\sum_{j \in S_{i}^{l}} \widehat{q}_{i j}^{l}, i \in N, l \in L .
\end{gathered}
$$

Linear programming formulation of the problem
The objective function (12) maximizes network lifetime (the sum of sink sojourn times at all possible locations), while (13) assures the non-negativity of $t_{l}, l \in L$. Multiplying the constraints on transmission rates (7) and the power of sensors (8) by $t_{l}$, gives (14) and (15) respectively. The left part of the inequality in (16) represents the total amount of energy consumed at sensor $i$ for transmitting and receiving data over all sojourn times of the sink at visited locations. Hence, the energy constraint in (16) simply states that the energy consumed at each sensor node $i$ should not exceed the initial energy $E_{i}$ of that sensor. Finally, multiplying the flow conservation condition (10) by $t_{l}$ gives (17).

The linear programming model above determines for every location $l \in L$ the duration $t_{l}$ for which the sink node stays at that place and the quantities $\widehat{q}_{i j}^{l}, i \in N, j \in S_{i}^{l}$, so that the network lifetime is maximized. The information transfer rates can also be computed as $q_{i j}^{l}=\widehat{q}_{i j}^{l} / t_{l}$ and, therefore, the model provides an optimal solution to both problems described earlier, the scheduling and the routing problem. If the optimal value for a $t_{l}$ is 0 , the sink does not visit location $l$. Every location $l \in L$ for which the optimal $t_{l}$ is positive, is visited by the sink for a time duration equal to $t_{l}$. The sink visiting order is not important since the traveling time of the sink between two locations is considered negligible and the information generation rate is independent of time.

A more general version of our linear programming formulation is to consider that while the sink $s$ is at a given location $l \in L$, the information transfer rates $q_{i j}^{l}, i \in N$, $j \in S_{i}^{l}$, can change. It may seem plausible that by modifying the rate $q_{i j}^{l}$ at which information is transmitted from sensor $i$ to its neighboring node $j$ during time $t_{l}$, the amounts of remaining battery energy of sensor nodes can be utilized more efficiently and, therefore, the duration of network operation can be prolonged. However, it is shown in the Appendix that this generalization of the problem does not result in any improvement for the overall lifetime of the network.

Note 2: The fact that the energy expenditures $e_{i j}^{T}, e_{i j}^{R}$ and the rates $q_{i j}^{l}$, are not restricted to a finite discrete set of values, implies an ideal power control sensor network. That is, each sensor is assumed to be able to adjust its transmission power accordingly, depending on the next hop node. This assumption is usually considered as an advantage, since it prevents a sensor from spending more power for transmission than the power that it actually needs. However, due to hardware constraints, in several practical systems there may be only little (a few power levels) power control. Taking this limitation into account is beyond the scope of current work. The problem becomes a discrete optimization one, and cannot be addressed directly by the proposed model. A trivial solution would be to solve the linear program and adjust the transmission power of each sensor to the minimum value that is larger than the one given by the model. However, a different approach is necessary in order to minimize the gap between theory and practice when taking the limitation of a finite set of discrete power levels into account. We also note that the model proposed here provides an upper bound on the performance of the system where each sensor can choose only among a discrete set of power levels. 


\section{NumERICAL RESUlts}

\section{A. Description of the Compared Models}

In this section we describe the models that we compare in order to evaluate numerically the performance of our linear programming formulation. Inside the parenthesis we give the abbreviation that we use for each model in the next section where we present the simulation results.

1) Linear Programming Model with Shortest Path Routing $(S P R)$ : This is a generalization of the approach followed in [23], so that it can be applied to general networks (the approach in [23] is restricted only to square grid networks with homogeneous sensors and no power control). The LP model determines only the sojourn times of the sink at every location $l \in L$ and, therefore, it provides a solution only to the scheduling problem described earlier. The routing problem is solved using a shortest path algorithm, where the cost of a path depends on the number of hops and the transmission energy requirement of each hop (in [23] there is no power control and the algorithm reduces to a minimum-number-ofhops routing algorithm). The shortest path algorithm used to route the packets to the sink does not take into account the remaining energy of the sensors, thus resulting in an overall network lifetime which is not optimal.

2) Linear Programming Model with Multiple Shortest Path Routing (MSPR): When there are multiple shortest paths, the authors in [23] select two of them and alternate the route between these two paths. Motivated by this variation, we modify the previous model so that the routing algorithm uses all existing shortest paths from a sensor to the sink. The LP model which determines sink sojourn times remains the same.

3) Linear Programming Model for the Static Sink case (Static Sink): When the sink remains static, we determine the lifetime achieved at every location $l \in L$ separately and select the one that gives the maximum value. Given the location that maximizes lifetime, the sink stays there until a sensor node runs out of battery energy for the first time and does not move to another place. For each location $l \in L$ separately we use the linear programming formulation given in Section III-B, replacing $L$ by $L^{\prime}=\{l\}$. Therefore, the Static Sink model maximizes lifetime for every location, but it is not optimal for the overall objective which is the sum of the sink sojourn times at all possible locations.

4) Optimal Linear Programming Formulation (LP-opt): This is the LP model proposed in Section III-B which provides the optimal solution to the scheduling and the routing problem and gives the best achievable overall network lifetime.

\section{B. Performance Evaluation Through Simulations}

In this section we compare numerically for various networks with different sizes and sink node placements the performance of the four models previously described. The simulations that follow attempt to model the following physical environment in a wireless sensor network. Assume that the sensor nodes are randomly deployed on a terrain where there are various obstacles that may prohibit direct communication of certain sensors. Assume also that the sink can be located in certain
$(0,100)$

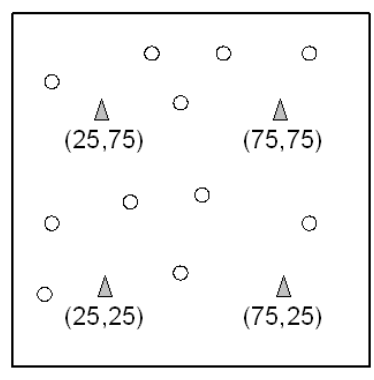

Scenario 1

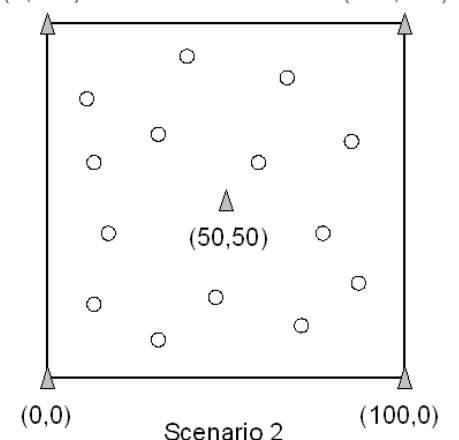

$\triangle$ Sink node location

- Sensor node
Fig. 2. Sensor nodes are randomly deployed in the network and two different sink node placement scenarios are examined.

places inside the terrain (not necessarily co-located with the sensor nodes) and that, for a given location, the sink is not within the transmission range of every sensor. That is, multihop routing may be needed in order to transfer the information from a sensor to the sink. We would like to evaluate the performance of the models in such an environment.

The figures that follow represent the averages of the results obtained from 100 randomly generated network instances for each network size considered. We generate random networks with a specified number of sensor nodes $(20,40, \ldots, 100)$ as follows. We fix a square grid of $100 \times 100$ points. A number of these points is randomly selected with uniform probability to represent the sensor nodes of the network. Note that the placement of the sensors does not form a grid network, but models a random deployment of the sensors on the terrain. The transmission energy consumed at sensor $i$ to transmit a data unit to its neighboring sensor $j$ depends on the distance $d_{(i, j)}$ between the two sensors and is given by $e_{i j}^{T}=d_{(i, j)}^{2}$. For simplicity in performing the experiments, we assume that the energy consumed by the receiver $j$ is small and thus can be neglected. Note that this assumption is not essential, since the reception cost is included in our LP formulation, and the qualitative performance of the compared models is not affected intensely. For each network instance there is a constraint on the maximum transmission energy expenditure among sensors, $e_{\max }^{T}$, which is defined as the smallest value that guarantees connectivity among the sensor nodes. Hence, a sensor node $j$ is a neighbor of $i$ if $e_{i j}^{T} \leq e_{\max }^{T}$. This constraint results in sparsely connected networks where multi-hop routing is necessary among the sensor nodes.

We investigate two different scenarios for the placement of the sink (see Fig. 2). In the first one the coordinates of possible sink locations are $(25,25),(25,75),(75,25),(75,75)$. That is, we split the grid to four quarters and, for a given location, the sink is within the transmission range only of the sensors that lie on the corresponding quarter of the grid. In the second scenario the sink can be placed at the four corners and at the center of the grid, that is, the coordinates of possible sink locations are $(0,0),(0,100),(100,0),(100,100),(50,50)$. When the 


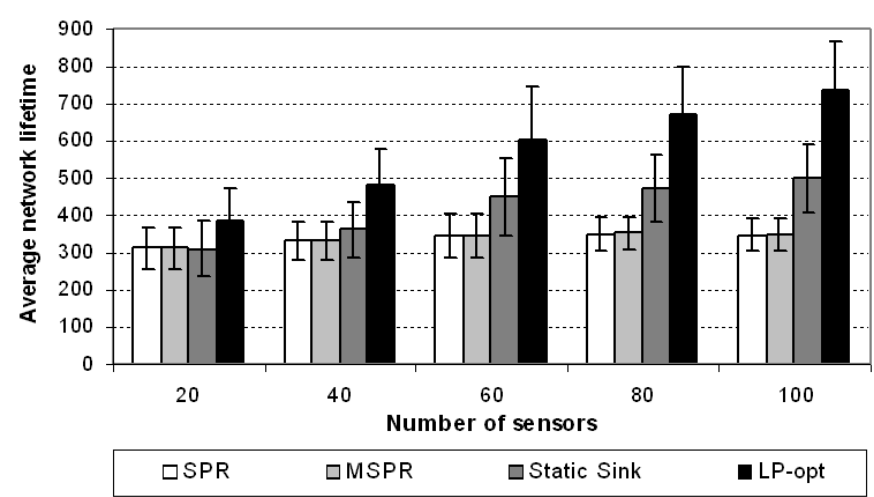

Fig. 3. Average lifetime (over all network instances) for various network sizes, sink locations $(25,25),(25,75),(75,25),(75,75)$.

sink is located at one of the four corners, it can be reached by the sensors that lie on the corresponding quarter of the grid as before. When it is placed at the center, it is within the transmission range of the sensors that lie on the area $(25,25)$, $(25,75),(75,25),(75,75)$. In any case, the energy required for transmission of a data unit from a sensor $i$ to the sink $s$ is given by $e_{i s}^{T}=d_{(i, s)}^{2}$. The rest of network parameters are set as follows: $Q_{i}=1$ and $E_{i}=10^{6}$ for every sensor node $i \in N$. We set no additional link capacity constraints $q_{i j}^{\max }$ and power constraints $P_{i}$, since the existence of $e_{\max }^{T}$ and the fact that $Q_{i}$ is relatively small guarantee that sensors will not consume excessive power for data transmissions. The LP models are solved for a given set of parameters by using LINGO [25].

Regarding the simulation environment, we make no claim of practical implementation of a real system. The specific energy consumption model used for the experiments and the values assigned to network parameters may not correspond to a real network. However, this does not invalidate our problem formulation nor the proposed model. Certainly, a different simulation environment will affect quantitatively the presented numerical results. Nevertheless, the qualitative performance of the compared algorithms is not expected to change much. Our objective is to use our model in order to calculate the optimal network lifetime, to obtain insight on the various design issues, and to compare the optimal solution to those obtained by using suboptimal heuristics.

The main performance metric of interest is the duration of network operation until a sensor node "dies" for the first time. Figures 3 and 4 show for the two scenarios considered the average lifetime obtained by each model over the 100 network instances created for each network size. The symbols ${ }_{\perp}^{\top}$ on top of each bar represent the corresponding standard deviations. We observe that our LP-opt model performs significantly better than the other three models for all networks examined. The lifetime achieved by LP-opt in Fig. 3 is $23.5 \%$ higher than that of SPR and $23.8 \%$ higher than that of Static Sink for $|N|=20$, while these percentages rise to $111.9 \%$ and $47.2 \%$, respectively, for $|N|=100$. In Fig. 4 the lifetime achieved by LP-opt is $28.8 \%$ higher than that of SPR and $14 \%$ higher than that of Static Sink for $|N|=20$, while the corresponding

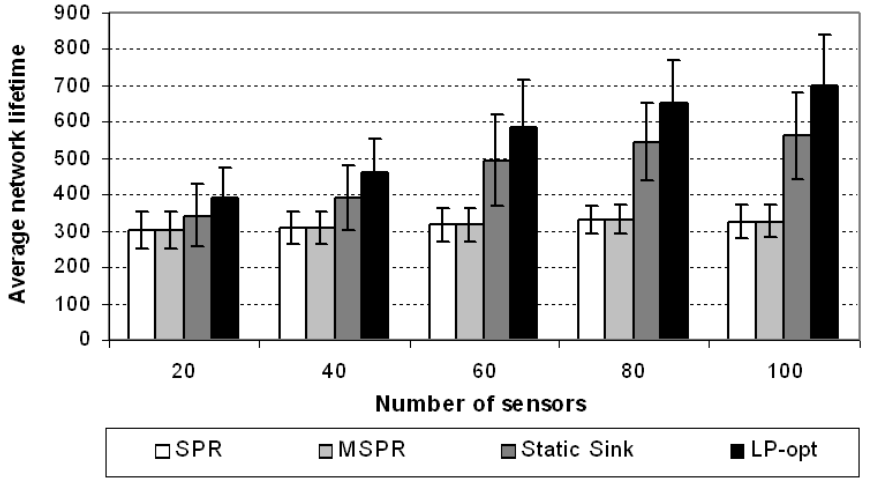

Fig. 4. Average lifetime (over all network instances) for various network sizes, sink locations $(0,0),(0,100),(100,0),(100,100),(50,50)$.

percentages for $|N|=100$ are $114.4 \%$ and $24.5 \%$. We see that in both figures the lifetime improvement ratio increases with the network size. As the network size increases, the density of sensor nodes (number of sensors per unit area) increases as well. Hence, there are more alternative paths which can be used by the LP-opt model to route the information from every sensor to the sink. This results in a more balanced energy depletion among the sensor nodes and, therefore, the lifetime achieved by LP-opt is considerably higher than the other three models. Note that the energy consumption model is the same for all network sizes and does not incorporate any additional energy costs for increased interference as the network size increases. Hence, larger networks appear to perform better in our simulations, which cannot be taken for granted in networks that operate in a high interference environment. In any case, the negative effect of larger interference induced by increasing the network size can be mitigated in part by the availability of more alternative paths provided by our model.

An interesting observation is that SPR and MSPR perform almost the same for all networks examined. This is due to the fact that the sensor nodes are randomly deployed inside the network and the transmission energy requirements depend on the distance between the communicating nodes. Hence, the costs of multiple paths that may exist from a sensor node to the sink are typically different and, most of the times, there exists only one shortest path to route the packets. A notable difference in the performance of these two models would appear in some special cases (e.g., grid networks). However, our aim is to evaluate the performance of the models in networks where the sensor nodes are realistically randomly placed in the sensor field. Another interesting observation is that Static Sink performs better than both SPR and MSPR. This is because the Static Sink model uses the optimal linear programming formulation of Section III-B and maximizes the lifetime for every location separately. Although the overall network lifetime achieved is shorter than LP-opt, since the sensor nodes close to the sink always relay the packets of all other sensors which drains them of their energy quite faster, it is still higher than that of SPR and MSPR which leave the routing problem outside the linear programming formulation (using a shortest path algorithm which is not optimal). 


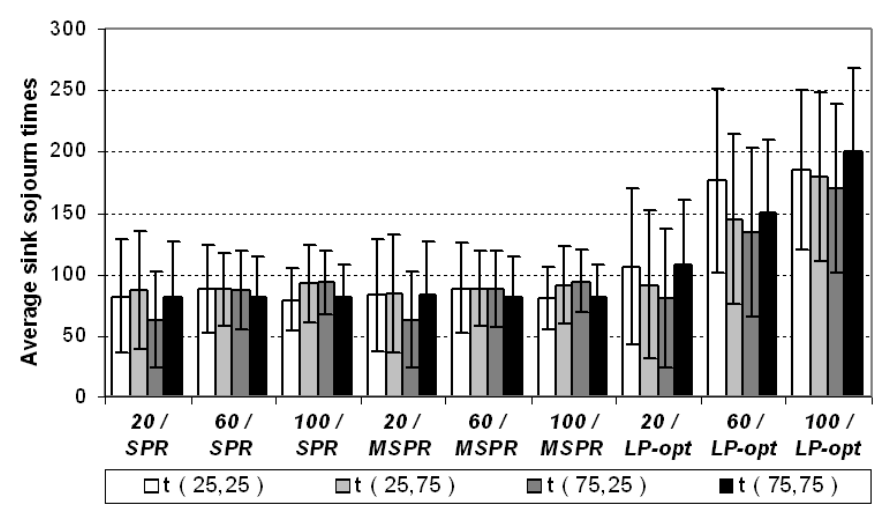

Fig. 5. Average sink sojourn times (over all network instances) for various network sizes at locations $(25,25),(25,75),(75,25),(75,75)$.

Figures 5 and 6 present the average sink sojourn times at the locations specified by the two scenarios over the 100 network instances created for each network size (we present the results obtained from networks with 20,60,100, sensor nodes). The models compared in these figures are SPR, MSPR, and LP-opt, since the lifetime achieved by Static Sink corresponds only to one location (the sink node stays in that location for the whole network operation and does not move to another place). It is worth noticing that the time spent by the sink is almost the same for every location in Fig. 5, while in Fig. 6 the sink node stays most of the time at location $(50,50)$ (center of network) and considerably less at the four corners. This observation is explained as follows. Averaging the results obtained from 100 randomly generated network instances for each network size, is almost the same as if we created a network with nearly uniform deployment of the sensor nodes. In Fig. 5, the sink node placements (centers of the four quarters of the network) are also uniform with respect to the deployment of the sensors. Hence, the sink spends almost equal time at every possible location. However, in Fig. 6, the sink locations are not uniform with respect to the sensors' deployment (there are more sensors around the center of the network than near the four corners of the area). Therefore, the sink node stays considerably more time at location $(50,50)$, since there are a lot of sensors close to it which can be used to relay the packets of all other sensors and prolong the overall network lifetime.

The fact that our approach results in a fair balancing of the energy depletion among the sensor nodes, compared to the other models, thus increasing network lifetime, can be justified by the results presented in Tables II and III. The models compared in these tables are SPR, Static Sink, and LP-opt, since MSPR performs almost the same as SPR as discussed earlier. Let $E_{i}^{\prime}$ be the residual energy of sensor node $i \in N$ at the end of network operation. For each network instance, we compute the percentages of sensors $i$ whose residual energy satisfies the following relations: $E_{i}^{\prime}=0, E_{i}^{\prime}<0.25 E_{i}$, $E_{i}^{\prime}<0.5 E_{i}, E_{i}^{\prime}<0.75 E_{i}$ (recall that $E_{i}$ is the initial battery energy of sensor $i$ ). We then average the corresponding percentages over the 100 network instances created for each network size considered. By expressing the residual energy of

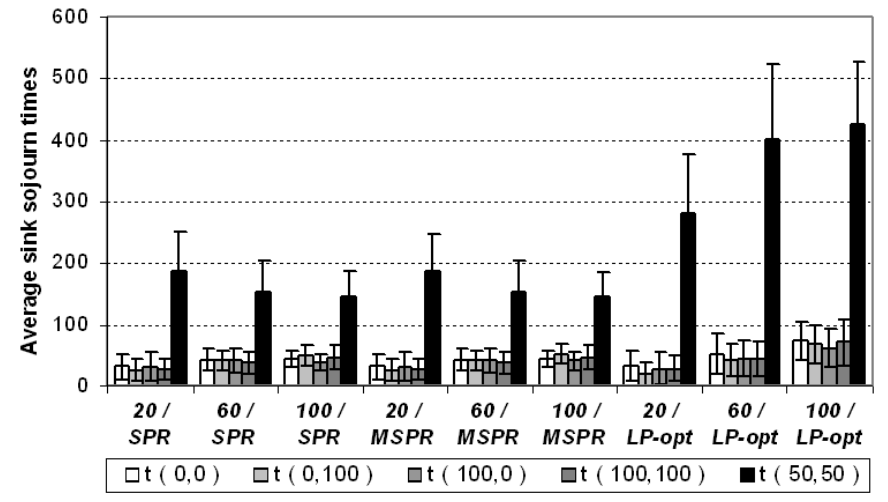

Fig. 6. Average sink sojourn times (over all network instances) for various network sizes at locations $(0,0),(0,100),(100,0),(100,100),(50,50)$.

every sensor as a fraction of its initial amount of battery energy, these percentages provide an indication about the distribution of the sensors' residual energies. For example, when there is a high percentage of sensors whose residual energy is zero, it means that the sensors' initial amounts of battery energy have been utilized efficiently and the energy is more evenly consumed among the sensor nodes. Hence, higher percentages of sensors with little residual energy, usually imply a higher overall network lifetime.

We observe that in both tables, LP-opt achieves much higher percentages of sensor nodes with little (or even zero) residual energy. We also see once more that Static Sink performs better than SPR, since the corresponding percentages are higher in all cases. SPR provides the worst performance of the three models compared, since there are many sensors at the end of network operation with high amounts of residual energy that have not been utilized to increase overall network lifetime. An interesting observation is that, as the network size increases, the percentages for LP-opt increase, while the corresponding percentages for SPR decrease. In Table III for example, the percentages of LP-opt for $E_{i}^{\prime}=0$ are $47 \%$ for $|N|=20$ and $70 \%$ for $|N|=100$. The corresponding percentages of SPR are $15 \%$ for $|N|=20$ and $4 \%$ for $|N|=100$. This behavior can be explained by the fact that the optimal performance of LPopt can be more easily observed in larger networks, while the non-optimal performance of SPR also becomes more obvious as the network size increases.

\section{DISCUSSION - ISSUES FOR FURTHER STUdy}

In this section we present some preliminary thoughts that could be used in the design of new heuristic algorithms for the problem discussed in this paper. The model that we propose determines the optimal sink movement patterns and sojourn times at different locations, and the optimal rates at which the sensed data must be transmitted from one sensor to another in order to be routed to the sink. However, implementing this model implies the need of a central controller where the solution to the LP problem will be computed. Also, the information obtained by solving the LP problem must be flooded to the network, so that every sensor is aware of the 
TABLE II

SCENARIO 1 - AVERAGE PERCENTAGES OF SENSOR NODES (OVER ALL NETWORK INSTANCES) FOR VARIOUS NETWORK SIZES, WHOSE RESIDUAL ENERGY SATISFIES THE FOLLOWING RELATIONS

\begin{tabular}{|c|c|c|c|c|c|c|c|c|c|c|c|c|}
\hline \multirow[b]{2}{*}{$|N|$} & \multicolumn{3}{|c|}{$E_{i}^{\prime}=0$} & \multicolumn{3}{|c|}{$E_{i}^{\prime}<0.25 \cdot E_{i}$} & \multicolumn{3}{|c|}{$E_{i}^{\prime}<0.5 \cdot E_{i}$} & \multicolumn{3}{|c|}{$E_{i}^{\prime}<0.75 \cdot E_{i}$} \\
\hline & SPR & Static Sink & LP-opt & SPR & Static Sink & LP-opt & SPR & Static Sink & LP-opt & SPR & Static Sink & LP-opt \\
\hline 20 & $13 \%$ & $22 \%$ & $36 \%$ & $21 \%$ & $28 \%$ & $43 \%$ & $29 \%$ & $36 \%$ & $51 \%$ & $47 \%$ & $52 \%$ & $67 \%$ \\
\hline 40 & $8 \%$ & $25 \%$ & $42 \%$ & $13 \%$ & $29 \%$ & $47 \%$ & $22 \%$ & $37 \%$ & $55 \%$ & $39 \%$ & $50 \%$ & $68 \%$ \\
\hline 60 & $6 \%$ & $32 \%$ & $52 \%$ & $10 \%$ & $36 \%$ & $55 \%$ & $18 \%$ & $42 \%$ & $62 \%$ & $35 \%$ & $54 \%$ & $73 \%$ \\
\hline 80 & $5 \%$ & $31 \%$ & $53 \%$ & $8 \%$ & $35 \%$ & $57 \%$ & $15 \%$ & $41 \%$ & $63 \%$ & $31 \%$ & $53 \%$ & $74 \%$ \\
\hline 100 & $4 \%$ & $31 \%$ & $56 \%$ & $7 \%$ & $35 \%$ & $60 \%$ & $12 \%$ & $41 \%$ & $65 \%$ & $27 \%$ & $52 \%$ & $75 \%$ \\
\hline
\end{tabular}

TABLE III

SCENARIO 2 - AVERAgE PERCENTAGES OF SENSOR NODES (OVER ALL NETWORK INSTANCES) FOR VARIOUS NETWORK SIZES, WHOSE RESIDUAL ENERGY SATISFIES THE FOLLOWING RELATIONS

\begin{tabular}{|c|c|c|c|c|c|c|c|c|c|c|c|c|}
\hline \multirow[b]{2}{*}{$|N|$} & \multicolumn{3}{|c|}{$E_{i}^{\prime}=0$} & \multicolumn{3}{|c|}{$E_{i}^{\prime}<0.25 \cdot E_{i}$} & \multicolumn{3}{|c|}{$E_{i}^{\prime}<0.5 \cdot E_{i}$} & \multicolumn{3}{|c|}{$E_{i}^{\prime}<0.75 \cdot E_{i}$} \\
\hline & SPR & Static Sink & LP-opt & SPR & Static Sink & LP-opt & SPR & Static Sink & LP-opt & SPR & Static Sink & LP-opt \\
\hline 20 & $15 \%$ & $25 \%$ & $47 \%$ & $22 \%$ & $32 \%$ & $52 \%$ & $32 \%$ & $41 \%$ & $61 \%$ & $55 \%$ & $58 \%$ & $75 \%$ \\
\hline 40 & $9 \%$ & $27 \%$ & $54 \%$ & $15 \%$ & $31 \%$ & $59 \%$ & $25 \%$ & $39 \%$ & $65 \%$ & $46 \%$ & $52 \%$ & $75 \%$ \\
\hline 60 & $7 \%$ & $32 \%$ & $63 \%$ & $11 \%$ & $36 \%$ & $66 \%$ & $20 \%$ & $43 \%$ & $70 \%$ & $41 \%$ & $54 \%$ & $78 \%$ \\
\hline 80 & $5 \%$ & $31 \%$ & $68 \%$ & $10 \%$ & $35 \%$ & $71 \%$ & $18 \%$ & $41 \%$ & $75 \%$ & $36 \%$ & $52 \%$ & $80 \%$ \\
\hline 100 & $4 \%$ & $31 \%$ & $70 \%$ & $8 \%$ & $34 \%$ & $73 \%$ & $16 \%$ & $40 \%$ & $76 \%$ & $34 \%$ & $50 \%$ & $81 \%$ \\
\hline
\end{tabular}

sink sojourn times at different places and of the rate at which it has to transmit data to its neighboring nodes. We note that the suboptimal algorithms proposed in previous work, described in Section IV-A, are also centralized. In our setup, we assume constant information generation rates (not necessarily the same for all sensors), while the previous approaches assume the same constant information generation rate for every sensor node.

An interesting issue for further study is the implementation of our model in a distributed environment where the sink sojourn times and the information transfer rates are not determined by a central node (possibly the sink). Distributed maximum lifetime routing algorithms for the case of fixed sink node location exist in the literature [26], but cannot be easily generalized to address the mobile sink problem.

Another interesting issue for future research is to develop an on-line algorithm which can be used in an adaptive environment, where the sensor nodes do not know the schedule of the sink in advance and the information generation process is arbitrary for every sensor. Such an algorithm could be as follows. We can use one of the energy-aware routing algorithms referenced in Section II for a given location of the sink. After an update interval, the sink node decides whether to stay at its current location or move to another place. The remaining lifetimes of the sensors can be used by the routing algorithm to determine new paths to the sink, so that the bottleneck nodes are avoided and a more balanced energy depletion among the sensor nodes is achieved. Such an algorithm would be greedy in a sense that the sink does not decide its schedule in advance, but moves from one location to another determining on-line the time to spend in every location according to the remaining lifetimes of the sensors. While this approach seems plausible, many issues have to be addressed for a complete description. Moreover, a careful evaluation must be performed in order to make sure that the overall approach works. We note that the optimal algorithm proposed in this work can be used as a yardstick against which one can compare the performance of new algorithms that might be proposed in the future to address the above-mentioned problems.

Other directions for further study include taking into account MAC layer issues and limitations in power control, i.e., considering only a few discrete power levels for the sensors. This constraint converts the problem to a discrete optimization one and a different approach is necessary in order to address the new issues that arise.

\section{CONCLUSiONS}

In this paper we addressed the problem of maximizing the lifetime in a wireless sensor network where the information generated by the monitoring sensor nodes needs to be routed efficiently to a mobile sink. The sink node can move to different places during network operation and the routing of the sensed data is performed across multiple hops with different transmission energy requirements. We exploited the capability of the sink to be mobile and presented a linear programming formulation that maximizes network lifetime. We showed that maximum lifetime can be achieved by solving optimally the joint problems of scheduling, which determines the sink sojourn times at different locations, and routing, which provides the appropriate energy-efficient paths to the sink. We compared numerically through simulations the performance of our model, which gives the best achievable network lifetime, 
with the case of static sink and with previously proposed models that focus mainly on the sink movement patterns and sojourn times, leaving the routing problem outside the linear programming formulation. Our approach maximizes network lifetime and results in a fair balancing of the energy depletion among the sensor nodes. The optimal lifetime provided by our model can be used as a performance measure in order to test the efficiency of other algorithms. Finally, we discussed the issue of proposing new heuristic on-line algorithms to be used in an adaptive and distributed environment where the sink sojourn times are not determined a priori.

\section{APPENDIX}

Here, we consider a more general version of the linear programming formulation given in Section III-B, where the information transfer rates $q_{i j}^{l}, i \in N, j \in S_{i}^{l}$, can change during time $t_{l}, l \in L$. Assume that each time duration $t_{l}$ is divided into a set of sub-intervals $t_{l}^{m}, m \in M_{l}$, such that for every location $l \in L$ it holds

$$
\sum_{m \in M_{l}} t_{l}^{m}=t_{l}
$$

Let also $q_{i j}^{l, m}$ be the rate at which information is transmitted from sensor $i$ to its neighboring node $j$ during time $t_{l}^{m}$. By defining $\hat{q}_{i j}^{l, m}=q_{i j}^{l, m} t_{l}^{m}$ as the amount of information transmitted from sensor $i$ to node $j$ during time $t_{l}^{m}$, the optimization problem becomes:

$$
\begin{gathered}
\text { Maximize } \sum_{l \in L} \sum_{m \in M_{l}} t_{l}^{m} \text { subject to } \\
t_{l}^{m} \geq 0, l \in L, m \in M_{l}, \\
0 \leq \widehat{q}_{i j}^{l, m} \leq q_{i j}^{\max } t_{l}^{m}, i \in N, j \in S_{i}^{l}, l \in L, m \in M_{l}, \\
\sum_{j \in S_{i}^{l} m \in M_{l}} \sum_{i j}^{T} \hat{q}_{i j}^{l, m}+\sum_{j: i \in S_{j}^{l} m \in M_{l}} \sum_{j i}^{R} \widehat{q}_{j i}^{l, m} \leq P_{i} \sum_{m \in M_{l}} t_{l}^{m}, \quad l \in N, \quad l \in L, \\
\sum_{l \in L} \sum_{j \in S_{i}^{l} m} \sum_{m \in M_{l}} e_{i j}^{T} \widehat{q}_{i j}^{l, m}+\sum_{l \in L} \sum_{j: i \in S_{j}^{l}, m \in M_{l}} \sum_{j i}^{R} \widehat{q}_{j i}^{l, m} \leq E_{i}, i \in N, \\
\sum_{j: i \in S_{j}^{l}} \hat{q}_{j i}^{l, m}+Q_{i} t_{l}^{m}=\sum_{j \in S_{i}^{l}} \widehat{q}_{i j}^{l, m}, i \in N, l \in L, m \in M_{l} .
\end{gathered}
$$

Define now the total amount of information transmitted from sensor $i$ to its neighbor $j$ during time $t_{l}$ as

$$
\widehat{q}_{i j}^{l}=\sum_{m \in M_{l}} \hat{q}_{i j}^{l, m}
$$

or, equivalently, the average rate at which information is transmitted from $i$ to $j$ during time $t_{l}$ as

$$
q_{i j}^{l}=\frac{\sum_{m \in M_{l}} q_{i j}^{l, m} t_{l}^{m}}{\sum_{m \in M_{l}} t_{l}^{m}} .
$$

By using these definitions and the definition of sub-intervals $t_{l}^{m}$, the optimization problem as stated above reduces to the linear programming formulation presented in Section IIIB. Therefore, it does not result in an improvement for our objective which is to maximize the overall network lifetime. However, such an approach might be useful if the information transfer rates $q_{i j}^{l}, i \in N, j \in S_{i}^{l}$, are allowed to change during time $t_{l}, l \in L$.

\section{REFERENCES}

[1] I. Chlamtac, M. Conti, and J.J.-N. Liu, "Mobile ad hoc networking: imperatives and challenges,” Ad Hoc Networks, vol. 1, no. 1, July 2003, pp. 13-64.

[2] A.J. Goldsmith and S.B. Wicker, "Design challenges for energyconstrained ad hoc wireless networks," IEEE Wireless Communications, vol. 9, no. 4, Aug. 2002, pp. 8-27.

[3] I.F. Akyildiz et al., "Wireless sensor networks: a survey," Computer Networks, vol. 38, no. 4, March 2002, pp. 393-422.

[4] S. Tilak, N.B. Abu-Ghazaleh, and W. Heinzelman, "A taxonomy of wireless micro-sensor network models," Mobile Computing and Communications Review, vol. 6, no. 2, Apr. 2002, pp. 28-36.

[5] I.F. Akyildiz and I.H. Kasimoglu, "Wireless sensor and actor networks: research challenges,” Ad Hoc Networks, vol. 2, no. 4, Oct. 2004, pp. 351-367.

[6] J.-H. Chang and L. Tassiulas, "Routing for maximum system lifetime in wireless ad-hoc networks," in Proc. of $37^{\text {th }}$ Annual Allerton Conf. on Communication, Control, and Computing, Monticello, IL, Sept.1999.

[7] J.-H. Chang and L. Tassiulas, "Energy conserving routing in wireless ad-hoc networks,” in Proc. of IEEE INFOCOM, Tel-Aviv, Israel, March 2000, pp. 22-31.

[8] J.-H. Chang and L. Tassiulas, "Maximum lifetime routing in wireless sensor networks,” in Proc. of ATIRP, College Park, MD, March 2000.

[9] J.-H. Chang and L. Tassiulas, "Maximum lifetime routing in wireless sensor networks,” IEEE/ACM Trans. on Networking, vol. 12, no. 4, Aug. 2004, pp. 609-619.

[10] K. Kalpakis, K. Dasgupta, and P. Namjoshi, "Efficient algorithms for maximum lifetime data gathering and aggregation in wireless sensor networks,” Computer Networks, vol. 42, no. 6, Aug. 2003, pp. 697-716.

[11] Y.T. Hou, Y. Shi, and H.D. Sherali, "Rate allocation in wireless sensor networks with network lifetime requirement," in Proc. of ACM MobiHoc, Roppongi, Japan, May 2004, pp. 67-77.

[12] H.-W. Yoon et al., "Energy efficient routing with power management to increase network lifetime in sensor networks," in Proc. of ICCSA, Assisi, Italy, May 2004, LNCS 3046, pp. 46-55.

[13] K. Akkaya and M. Younis, "Energy-aware delay-constrained routing in wireless sensor networks,” Int. J. of Communication Systems, vol. 17, no. 6, Aug. 2004, pp. 663-687.

[14] L. Zhang et al., "A fair energy conserving routing algorithm for wireless sensor networks," in Proc. of EUSAI, Eindhoven, The Netherlands, Nov. 2004, LNCS 3295, pp. 303-314.

[15] A. Bogdanov, E. Maneva, and S. Riesenfeld, "Power-aware base station positioning for sensor networks," in Proc. of IEEE INFOCOM, Hong Kong, March 2004.

[16] E.I. Oyman and C. Ersoy, "Multiple sink network design problem in large scale wireless sensor networks," in Proc. of ICC, Paris, France, June 2004.

[17] A. Chakrabarti, A. Sabharwal, and B. Aazhang, "Using predictable observer mobility for power efficient design of sensor networks," in Proc. of IPSN, Palo Alto, CA, Apr. 2003, LNCS 2634, pp. 129-145.

[18] H.S. Kim, T.F. Abdelzaher, and W.H. Kwon, "Minimum-energy asynchronous dissemination to mobile sinks in wireless sensor networks," in Proc. of ACM SenSys, Los Angeles, CA, Nov. 2003, pp. 193-204.

[19] S.R. Gandham et al., "Energy efficient schemes for wireless sensor networks with multiple mobile base stations," in Proc. of IEEE GLOBECOM, San Francisco, CA, Dec. 2003, pp. 377-381.

[20] R. Urgaonkar and B. Krishnamachari, "FLOW: An efficient forwarding scheme to mobile sink in wireless sensor networks," in Poster of IEEE SECON, Santa Clara, CA, Oct. 2004.

[21] P. Baruah, R. Urgaonkar, and B. Krishnamachari, "Learning-enforced time domain routing to mobile sinks in wireless sensor fields," in Proc. of IEEE EmNetS-I, Tampa, FL, Nov. 2004.

[22] Y. Tirta et al., "Efficient collection of sensor data in remote fields using mobile collectors,” in Proc. of ICCCN, Chicago, IL, Oct. 2004. 
[23] Z.M. Wang et al., "Exploiting sink mobility for maximizing sensor networks lifetime," in Proc. of HICSS, Big Island, Hawaii, Jan. 2005.

[24] S.G. Nash and A. Sofer, Linear and Nonlinear Programming, New York: McGraw-Hill, 1996.

[25] Lindo Systems Inc., http://www.lindo.com .

[26] R. Madan and S. Lall, "Distributed algorithms for maximum lifetime routing in wireless sensor networks," in Proc. of IEEE GLOBECOM, Dallas, TX, Nov.-Dec. 2004.

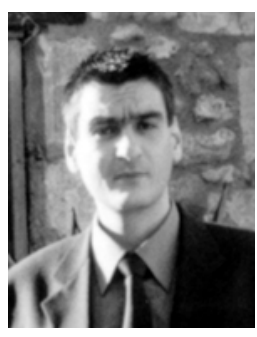

Ioannis Papadimitriou was born in Veria, Greece, in 1976. He received his five year Diploma from the Department of Electronic and Computer Engineering, Technical University of Crete (Chania), Greece, in 1999 (graduating 2nd in class). He is currently a postgraduate student - Ph.D. candidate at the Telecommunications division, Department of Electrical and Computer Engineering, Aristotle University of Thessaloniki, Greece. His doctoral thesis deals with the design of wireless ad hoc networks. His research interests include broadcast and multicast communication, energy conservation, routing and topology control protocols, MAC layer and QoS issues. During his studies he has been honored with awards and scholarships by the Technical University of Crete, the Hellenic Telecommunications Organization S.A. (OTE S.A.) and Ericsson Hellas S.A. Mr. Papadimitriou has been a member of the Technical Chamber of Greece (TEE) since March 2000, and he has been supported by the Public Benefit Foundation "ALEXANDER S. ONASSIS", Athens, Greece, with a scholarship for his doctoral studies from October 2001 to March 2005.

E-mail: ipapad@egnatia.ee.auth.gr

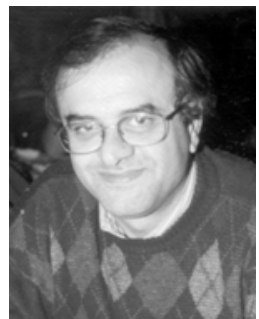

Leonidas Georgiadis received the Diploma degree in electrical engineering from Aristotle University, Thessaloniki, Greece, in 1979, and his M.S. and Ph.D. degrees both in electrical engineering from the University of Connecticut, in 1981 and 1986, respectively. From 1981 to 1983 he was with the Greek army. From 1986 to 1987 he was Research Assistant Professor at the University of Virginia, Charlottesville. In 1987 he joined IBM T.J. Watson Research Center, Yorktown Heights, as a Research Staff Member. Since October 1995, he has been with the Telecommunications Department of Aristotle University, Thessaloniki, Greece. His interests are in the area of wireless networks, high speed networks, distributed systems, routing, scheduling, congestion control, modeling and performance analysis. Prof. Georgiadis is a senior member of IEEE Communications Society. In 1992 he received the IBM Outstanding Innovation Award for his work on goal-oriented workload management for multi-class systems.

E-mail: leonid@auth.gr 